\title{
Three-Dimensional Stress Concentration Around a Cylindrical Hole in a Semi-Infinite Elastic Body'
}

\section{E. STERNBERG}

Division of Engineering and Applied Science,

California Institute of Technology, Pasadena, Calif.
This paper contains a three-dimensional solution, exact within classical elastostatics, for the stresses and deformations arising in a half-space with a semi-infinite transverse cylindrical hole, if the body-at infinite distances from its cylindrical boundary-is subjected to an arbitrary uniform plane field of stress that is parallel to the bounding plane. The solution presented is in integral form and is deduced with the aid of the Papkovich stress functions by means of an especially adapted, unconventional, integral. transform technique. Numerical results for the nonvanishing stresses along the boundary of the hole and for the normal displacement at the plane boundary, corresponding to several values of Poisson's ratio, are also included. These results exhibit in detail the three-dimensional stress boundary layer that emerges near the edges of the hole in the analogous problem for a plate of finite thickness, as the ratio of the plate thickness to the diameter of the hole grows beyond bounds. The results obtained thus illustrate the limitations inherent in the two-dimensional plane-strain treatment of the spatial plane problem; in addition, they are relevant to failure considerations and of interest in connection with experimental stress analysis.

\section{Introduction: Motivation of This Investigation}

T

$\mathrm{T}_{\mathrm{H}}$ theory of homogeneous and isotropic elastic solids is in fact a three-dimensional boundary-value problem of formidable complexity. It consists of the determination of the displacements and stresses throughout an elastic body of cylindrical (or prismatic) shape if the surface tractions are confined to the lateral boundary, the terminal cross sections being free from tractions, provided the prescribed body forces and surface loads are applied at right angles to the generators of the lateral boundary and do not vary in a direction parallel to these generators. ${ }^{2}$

As is well known, the plane-strain and plane-stress solutions associated with the spatial plane problem satisfy the governing differential equations rigorously but meet all of the boundary conditions only in highly exceptional circumstanees: In general, they yield merely approximations to the desired three-dimensional solution, which are of different and limited applicability. The plane-strain solution conforms to the lateral boundary conditions but ordinarily fails to clear the ends of the cylinder from normal tractions. On the other hand, the plane-stress solution fulfills the requirement of traction-free ends but usua'ly violates the lateral boundary conditions, which in this instance are satisfied merely in the thickness mean. Finally, the associated generalized plane-stress solution furnishes the thickness averages of the desired displacements and stresses within the ordinarily approximative assumption that the axial normal stress vanishes identically. Further, these averages coincide with the corresponding

${ }^{1}$ This investigation was carried out under the auspices of the United States Atomic Energy Commission and was supported in part by the Office of Naval Research under Contract Nonr-220(58).

${ }^{2} \mathrm{We}$ are at present concerned exclusively with the second plane boundary-value problem, in which the surface tractions are preseribed over the entire lateral boundary.

Presented at the Fifth U. S. National Congress of Applied Mechanics, University of Minnesota, Minneapolis, Minn., June 14-17, 1966.

Discussion of this paper should be addressed to the Editorial Department, ASME, United Engineering Center, 345 East 47 th Street, New York, N. Y. 10017, and will be accepted until January 15, 1967. Discussion received after the closing date will be returned. Manuscript received by ASME Applied Mechanics Division, May 20, 1965; final draft, December 6, 1965. average displacements and stresses obtainable from the planestress solution.

In accordance with the preceding commonplace observations, in general neither the required displacement field nor its accompanying stress field is plane, and both fields vary from one cross section of the body to another. Exceptions to this statement arise if $(a)$ Poisson's ratio is zero or $(b)$ the axial normal stress predicted by the plane-strain solution is either constant or a linear function of rectangular Cartesian coordinates chosen within a representative cross section. In both of these special cases the plane-stress solution is the exact three-dimensional solution of the original problem. In case $(a)$ the exact solution to the plane problem is also identical with the associated plane-strain solution, whereas in case $(b)$ the stresses found in the plane-strain solution are exact, except possibly for the axial normal stress which here again vanishes identically in the actual solution. A particularly important example of case $(b)$ is supplied by the axisymmetric plane problem when there are no body forces present. In the absence of the degeneracies just described, the generalized plane-stress solution supplies a useful approximation to the desired stresses and deformations if the ratio of the length of the cylinder (thickness of the plate) to the relevant cross-sectional dimension is sufficiently small. In contrast, the plane-strain solution-modified, if necessary, by superposition of the solution corresponding to a uniaxial stress field so as to assure the selfequilibrance of the residual tractions on the ends of the cylinderyields an approximation appropriate to the central portion of a sufficiently long cylinder.

General methods for dealing with three-dimensional aspects of the plane problem have engaged the attention of several investigators. Thus Reissner $[1]^{3}$ (1942) proposed a semi-direct variational method aiming at three-dimensional corrections for the theory of generalized plane stress, applicable to relatively thin plates. Green [2] (1949), employing infinite series of exact solutions to the elastostatic field equations, developed a formal scheme for coping with a class of spatial boundary-value problems that includes the plane problem. Reiss and Locke [3] (1961), motivated by the objectives of [1] and using the generalized planestress solution as a zero-order approximation, pursued the determination of the desired corrections on the basis of a formal expansion of the stress field in powers of the thickness parameter; in

${ }^{3}$ Numbers in brackets designate References at end of paper. 
this connection, they adopted a boundary-layer technique originally devised by Friedrichs [4] (1949) for the analogous plate flexure problem. The possibility of establishing successive approximations to the exact solution of the plane problem with the aid of expansions in powers of Poisson's ratio-using the planestrain solution as the zero-order approximation-was considered and found impractical by Sternberg and Muki [5] (1959).

As far as the three-dimensional treatment of specific plane problems is concerned, most efforts have been directed at the problem presented by the stress concentration around a transverse circular cylindrical hole in an infinite slab under uniform loads at infinity - a problem of particular engineering interest. ${ }^{4}$ The well-known corresponding two-dimensional solutions are due to Kirsch [7] (1898). A three-dimensional solution, which is in infinite series form and whose structure is highly complicated, was deduced by Green [8] (1948) for the nontrivial case of a uniaxial loading at infinity ${ }^{5}$ prior to the publication of his general paper [2]. The solution arrived at in [8] satisfies the requisite differential equations rigorously; the accompanying boundary conditions are met to a satisfactory degree of approximation by means of an iterative procedure. A rather crude and quantitatively inadequate three-dimensional analysis of the same problem was carried out independently by Sternberg and Sadowsky [9] (1949), who employed a modification of the Ritz energy method. Alblas [10] (1957) returned to the problem under discussion. Applying the systematic scheme proposed by Green in [2], he succeeded in constructing a series representation of the three-dimensional solution which is more convenient than that contained in [8] and performed extensive numerical evaluations. ${ }^{6}$ Finally, Reiss [11] (1963) applied the expansion technique previously developed in [3] to the determination of three-dimensional corrections for the generalized plane-stress solution given in [7].

As is to be anticipated on intuitive grounds, and is confirmed by the numerical results presented in [9], [10], the distribution of the stresses across the plate thickness becomes increasingly more sensitive to changes in the thickness-ratio, i.e., the ratio of the plate thickness to the diameter of the hole, at comparatively large values of this ratio. As the thickness-ratio approaches infinity, the transverse variation of the stresses near the cylindrical boundary becomes progressively more pronounced and localized within boundary layers adjacent to the plate faces. Further, the foregoing three-dimensional boundary-layer effect is inadequately represented by the existing solutions to the problem at hand and requires separate treatment for its reliable quantitative appraisal. This leads one to inquire into the stresses and deformations in an elastic half-space with a semi-infinite transverse circular cylindrical hole, due to an arbitrary plane field of stress that is applied parallel to the bounding plane at infinite distances from the axis of the hole.

The solution to the half-space problem just described, which is our main objective, thus supplements the earlier three-dimensional results appropriate to the plate of finite thickness-ratio and supplies further insight into the limitations attached to the conventional two-dimensional treatment of plane problems. In addition, the present problem possesses a twofold intrinsic interest. First, a knowledge of the triaxiality inherent in the stress distribution near the edges of the hole is desirable from the point of view of failure considerations. Second, the three-dimensional

4 The only other plane problem that appears to have been so considered is that of an infinite slab subjected to an internal transverse line load of uniform intensity, for which Green and Willmore [6] (1948) deduced an exact solution in integral form. Although more easily tractable, this problem is less attractive as a vehicle for studying the limitations of the two-dimensional theory since it fails to involve a dimensionless thickness parameter; it is also less interesting from a physical point of view.

5 In the special instance of isotropic tension or compression at infinity, the plane-stress solution constitutes the exact three-dimensional solution. Cf. our earlier remark concerning the rotationally symmetric plane problem.

6 In [10], Alblas also dealt comprehensively with the analogous flexure problem, whose history is beyond our present scope. effects sought here, which are absent when Poisson's ratio vanishes, are bound to depend sensitively upon the value of this physical parameter; consequently, the results also illustrate the difficulties that may be encountered in the interpretation of experimental stress-analysis findings when Poisson's ratio of the model used differs appreciably from that of the prototype.

At first sight, the half-space problem to be treated presently might appear to be simpler-or, at least, no more complicatedthan its counterpart for the plate of finite thickness. This expectation appears to be unwarranted. In the plate problem ${ }^{7}$ it is possible, by means of the Papkovich stress functions and separation of variables, to construct a triply infinite aggregate of solutions to the governing differential equations, each member of which clears the plate faces from tractions and corresponds to vanishing loads at infinity. With the aid of appropriate Fourier expansions in the thickness coordinate, one may thus reduce the task of removing the residual tractions to which the associated plane-stress solution gives rise on the cylindrical boundary to the solution of a triply infinite system of linear algebraic equations for the unknown coefficients of superposition. Such an expansion scheme is no longer applicable when the range of the thickness coordinate is unbounded; nor is the half-space problem at hand amenable to a treatment by standard integral-transform techniques.

The method of solution adopted in this paper may be outlined briefly as follows. We first use the associated plane-strain solution to transform the original problem into one governed by prescribed normal tractions on the plane boundary and otherwise vanishing loads. To cope with this "residual problem of plane strain," we then employ the Papkovich stress functions to construct a solution in integral form to the elastostatic field equations that leaves the plane boundary free from shearing tractions, yields vanishing stresses at infinite distances from the hole, and involves four arbitrary weight functions of the integration parameter. Further, this solution, which is not obtainable by separation of variables alone, is so constructed as to insure that the application of the remaining four boundary conditions leads to a system of simultaneous integral equations for the unknown weight functions which is reducible to a single one-dimensional integral equation of Fredholm's second kind. The latter reduction is accomplished with the aid of the inversion theorem for the Fourier transform, as well as by recourse to an inversion formula that is closely related to Weber's integral theorem. ${ }^{8}$ In this manner, we arrive at an integral representation for the exact solution to the original problem in terms of the solution of the foregoing integral equation and otherwise involving only known functions.

The final integral equation was solved numerically on an electronic computer for values of Poisson's ratio of $1 / 4$ and $1 / 2$, the solution of the problem corresponding to zero Poisson's ratio being known beforehand. Unfortunately, the subsequent evaluation of the improper integrals for the desired stresses and displacements, as well as the numerical check on the boundary conditions, did not yield to routine numerical integration methods. To overcome the convergence difficulties encountered, it was essential to remove in closed form certain contributions to the required integrals that impede their convergence. This, in turn, necessitated a detailed examination of the asymptotic behavior of the integrands concerned.

As is suggested by the preceding remarks, the burden of the numerical analysis of the solution established was at least equal to the effort expended on its derivation. There is ample indication to suppose that the numerical results obtained are accurate well beyond the limits of physical relevance.

\footnotetext{
${ }^{7}$ See $[2],[10]$.

${ }^{8}$ See Watson [12], p. 468. A similar modification of Weber's theorem was applied by Blenkarn and Wilhoit [13] in connection with a related, axisymmetric, problem for the half-space with a cylindrical bore. In [13] the loading consists of a uniform band of pressure applied at the entrance of the hole.
} 


\section{Formulation of Problem: Reduction to a Residual Problem}

Let $\left(x_{1}, x_{2}, x_{3}\right)$ be rectangular Cartesian coordinates and $R$ the region of space characterized by

$$
0 \leq x_{3}<\infty, \quad r=\left(x_{1}^{2}+x_{2}^{2}\right)^{1 / 2} \geq a,
$$

so that $R$ is a half-space with a semi-infinite transverse circular cylindrical opening of radius $a$ (Fig. 1). Let $\Pi\left(x_{3}=0, a \leq r<\right.$ $\infty)$ be the plane portion of the boundary of $R$ and $\Gamma(r=a$, $0 \leq x_{3}<\infty$ ) its cylindrical part. Assume further that $R$ is occupied by a homogeneous and isotropic elastic solid with the shear modulus $\mu$ and Poisson's ratio $\nu$. With reference to the preceding choice of coordinates, and in the usual indicial notation, the problem to be considered admits the following formulation:

Throughout the interior of $R$ the Cartesian components of displacement and stress, $u_{i}$ and $\sigma_{i j}$, must satisfy the displacement equations of equilibrium

$$
u_{i, j i}+\frac{1}{1-2 \nu} u_{i, j i}=0
$$

together with the stress-displacement relations

$$
\sigma_{i j}=\mu\left(\frac{2 \nu}{1-2 \nu} \delta_{i j} u_{k, k}+u_{i, j}+u_{j, i}\right),
$$

in which $\delta_{i j}$ is the Kronecker delta. Since both $\Pi$ and $\Gamma$ are to be free from tractions, the boundary conditions take the form

$$
\sigma_{3 i}=0 \text { on } \Pi
$$

and

$$
\sigma_{i j} n_{j}=0 \text { on } \Gamma,
$$

provided $n_{j}$ denotes the components of the unit outward normal of $\Gamma$. Finally, as $r \rightarrow \infty$, the stress field is required to approach a uniform plane state of stress that is parallel to $\Pi$. Since, without loss in generality, the principal axes of this limiting state of stress may be assumed coincident with the coordinate axes, the preceding loading condition becomes

$$
\sigma_{11} \rightarrow \sigma_{1}, \sigma_{22} \rightarrow \sigma_{2}, \sigma_{12} \rightarrow 0, \quad \sigma_{3 i} \rightarrow 0 \text { as } r \rightarrow \infty,
$$

where $\sigma_{1}$ and $\sigma_{2}$ are prescribed constants.

The complete solution of (2) admits the well-known representation in terms of the Papkovich-Neuber stress functions, given by

$$
u_{i}=\frac{1}{2 \mu}\left[\left(\Phi+x_{j} \Psi_{j}\right)_{, i}-4(1-\nu) \Psi_{i}\right]
$$

with

$$
\nabla^{2} \Phi=0, \quad \nabla^{2} \Psi_{i}=0 .
$$

Further, from (3), (7), and (8) follows

$\sigma_{i j}=\Phi_{, i j}-(1-2 \nu)\left(\Psi_{i, j}+\Psi_{j, i}\right)+x_{k} \Psi_{k, i j}-2 \nu \delta_{i j} \Psi_{k, k}$.

Accordingly, the problem under consideration reduces to the determination of functions $\Phi$ and $\Psi_{i}$ that are harmonic in the interior of $R$ and such that the stresses (9) obey (4), (5), and (6).

Let $S$, with the Cartesian components $u_{i}$ and $\sigma_{i j}$, be the desired solution to the foregoing boundary-value problem. Further, let $S^{\prime}$ and $S^{\prime \prime}$ be the respective solutions of (2) to (6) appropriate to the following two basic loading cases:

Case 1.

$\sigma_{11} \rightarrow \sigma, \quad \sigma_{22} \rightarrow \sigma, \quad \sigma_{12} \rightarrow 0, \quad \sigma_{3 i} \rightarrow 0 \quad$ as $\quad r \rightarrow \infty$.

Case 2.

$\sigma_{11} \rightarrow \sigma, \quad \sigma_{22} \rightarrow-\sigma, \quad \sigma_{12} \rightarrow 0$,

$$
\sigma_{3 i} \rightarrow 0 \text { as } r \rightarrow \infty .
$$

Thus $S^{\prime}$ and $S^{\prime \prime}$ correspond, respectively, to a plane isotropic

- The body forces are assumed to vanish identically.

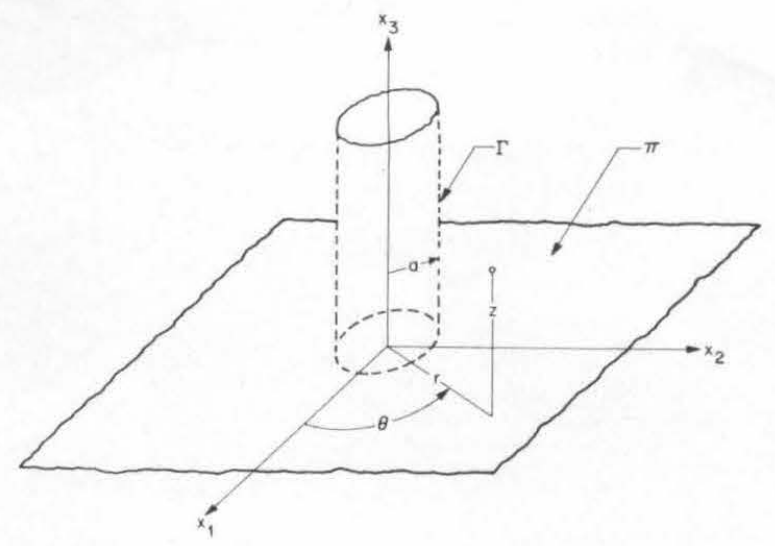

Fig. I Half-space with cylindrical hole, Cartesian and cylindrical coordinates

state of stress and to a plane state of pure shear at infinity. Then, clearly,

$$
S=\frac{\sigma_{1}+\sigma_{2}}{2 \sigma} S^{\prime}+\frac{\sigma_{1}-\sigma_{2}}{2 \sigma} S^{\prime \prime}
$$

The solution $S^{\prime}$ to the axisymmetric plane problem arising in Case 1 is elementary, as is the plane-strain solution associated with Case 2. Both of these familiar solutions will be recalled shortly.

We now introduce cylindrical coordinates $(r, \theta, z)$ through the mapping

$$
\begin{gathered}
x_{1}=r \cos \theta, \quad x_{2}=r \sin \theta, \quad x_{3}=z, \\
0 \leq r<\infty, \quad 0 \leq \theta<2 \pi, \quad-\infty<z<\infty,
\end{gathered}
$$

and, at the same time, define the dimensionless coordinates

$$
\rho=r / a, \quad \zeta=z / a,
$$

the dimensionless stress functions

$$
\hat{\Phi}=\Phi / a^{2} \sigma, \quad \hat{\Psi}_{i}=\Psi_{i} / a \sigma,
$$

as well as the dimensionless cylindrical components of displacement and stress

$$
\begin{aligned}
& v_{r}=2 \mu u_{r} / a \sigma, \quad v_{\theta}=2 \mu u_{\theta} / a \sigma, \quad v_{s}=2 \mu u_{z} / a \sigma, \\
& \tau_{r r}=\sigma_{r r} / \sigma, \quad \tau_{\theta \theta}=\sigma_{\theta \theta} / \sigma, \quad \tau_{z z}=\sigma_{z z} / \sigma, \\
& \tau_{\theta z}=\sigma_{\theta z} / \sigma, \quad \tau_{z r}=\sigma_{z} / \sigma, \quad \tau_{r \theta}=\sigma_{r \theta} / \sigma .
\end{aligned}
$$

With this notation, on setting

$$
\alpha=2(1-\nu), \quad \hat{\omega}=\hat{\Phi}+\rho \hat{\Psi}_{1} \cos \theta+\rho \hat{\Psi}_{2} \sin \theta+\zeta \hat{\Psi}_{3},
$$

equations (7) in cylindrical coordinates ${ }^{11}$ assume the form

$$
\begin{aligned}
& v_{r}=\frac{\partial \hat{\omega}}{\partial \rho}-2 \alpha\left(\hat{\Psi}_{1} \cos \theta+\hat{\Psi}_{2} \sin \theta\right), \\
& v_{\theta}=\frac{1}{\rho} \frac{\partial \hat{\omega}}{\partial \theta}+2 \alpha\left(\hat{\Psi}_{1} \sin \theta-\hat{\Psi}_{2} \cos \theta\right), \\
& v_{z}=\frac{\partial \hat{\omega}}{\partial \zeta}-2 \alpha \hat{\Psi}_{3} .
\end{aligned}
$$

Similarly, equations (9) give way to

${ }^{10}$ Here, $\sigma \neq 0$ is implied; we avoid the normalization $\sigma=1$ in order not to obscure the dimensionality of $S^{\prime}$ and $S^{\prime \prime}$. Addition and multiplication by a scalar constant of solutions to the elastostatic field equations are to be interpreted in the sense of the corresponding operations applied to their fields of displacement and stress.

11 See [14] for the Papkovich-Neuber solution in general orthogona] curvilinear coordinates. 


$$
\begin{aligned}
& \tau_{r}=\frac{\partial^{2} \hat{\omega}}{\partial \rho^{2}}+(\alpha-2)\left[-\frac{1}{\rho} \frac{\partial \hat{\Psi}_{1}}{\partial \theta} \sin \theta\right. \\
& \left.+\frac{1}{\rho} \frac{\partial \hat{\Psi}_{2}}{\partial \theta} \cos \theta+\frac{\partial \hat{\Psi}_{3}}{\partial \zeta}\right] \\
& -(\alpha+2)\left[\frac{\partial \hat{\Psi}_{1}}{\partial \rho} \cos \theta+\frac{\partial \hat{\Psi}_{2}}{\partial \rho} \sin \theta\right] \text {, } \\
& \tau_{\theta \theta}=\frac{1}{\rho^{2}} \frac{\partial^{2} \hat{\omega}}{\partial \theta^{2}}+\frac{1}{\rho} \frac{\partial \hat{\omega}}{\partial \rho} \\
& +(\alpha-2)\left[\frac{\partial \hat{\Psi}_{1}}{\partial \rho} \cos \theta+\frac{\partial \hat{\Psi}_{2}}{\partial \rho} \sin \theta+\frac{\partial \hat{\Psi}_{3}}{\partial \zeta}\right] \\
& +(\alpha+2)\left[\frac{1}{\rho} \frac{\partial \hat{\Psi}_{1}}{\partial \theta} \sin \theta-\frac{1}{\rho} \frac{\partial \hat{\Psi}_{2}}{\partial \theta} \cos \theta\right] \text {, } \\
& \tau_{z .}=\frac{\partial^{2} \hat{\omega}}{\partial \zeta^{2}}+(\alpha-2)\left[\frac{\partial \hat{\Psi}_{1}}{\partial \rho} \cos \theta+\frac{\partial \hat{\Psi}_{2}}{\partial \rho} \sin \theta\right. \\
& \left.-\frac{1}{\rho} \frac{\partial \hat{\Psi}_{1}}{\partial \theta} \sin \theta+\frac{1}{\rho} \frac{\partial \hat{\Psi}_{2}}{\partial \theta} \cos \theta\right]-(\alpha+2) \frac{\partial \hat{\Psi}_{3}}{\partial \zeta}, \\
& \tau_{\theta z}=\frac{1}{\rho} \frac{\partial^{2} \hat{\omega}}{\partial \theta \partial \zeta}+\alpha\left[\frac{\partial \hat{\Psi}_{1}}{\partial \zeta} \sin \theta-\frac{\partial \hat{\Psi}_{2}}{\partial \zeta} \cos \theta-\frac{1}{\rho} \frac{\partial \hat{\Psi}_{3}}{\partial \theta}\right], \\
& \tau_{z r}=\frac{\partial^{2} \hat{\omega}}{\partial \rho \partial \zeta}-\alpha\left[\frac{\partial \hat{\Psi}_{1}}{\partial \zeta} \cos \theta+\frac{\partial \hat{\Psi}_{2}}{\partial \zeta} \sin \theta+\frac{\partial \hat{\Psi}_{3}}{\partial \rho}\right], \\
& \tau_{r \theta}=\frac{1}{\rho} \frac{\partial^{2} \hat{\omega}}{\partial \rho \partial \theta}-\frac{1}{\rho^{2}} \frac{\partial \hat{\omega}}{\partial \theta}-\alpha\left[\frac{1}{\rho} \frac{\partial \hat{\Psi}_{1}}{\partial \theta} \cos \theta\right. \\
& \left.+\frac{1}{\rho} \frac{\partial \hat{\Psi}_{2}}{\partial \theta} \sin \theta-\frac{\partial \hat{\Psi}_{1}}{\partial \rho} \sin \theta+\frac{\partial \hat{\Psi}_{2}}{\partial \rho} \cos \theta\right] \text {. }
\end{aligned}
$$

In connection with (8), we cite the cylindrical form of the Laplacian operator

$$
a^{2} \nabla^{2}=\frac{\partial^{2}}{\partial \rho^{2}}+\frac{1}{\rho} \frac{\partial}{\partial \rho}+\frac{1}{\rho^{2}} \frac{\partial^{2}}{\partial \theta^{2}}+\frac{\partial^{2}}{\partial \zeta^{2}} .
$$

The plane-stress solution for Case 1 , which coincides with the exact solution $S^{\prime}$, and the plane-strain solution associated with Case 2, which will hereafter be designated by $S^{\prime \prime}$, may be generated with the aid of the Papkovich-Neuber stress functions. ${ }^{12}$ As is readily confirmed by means of (14)-(20), the results may be summarized as follows:

Solution $S^{\prime}$ (Plane-Stress Solution for Case 1).

$$
\begin{array}{cc}
\hat{\Phi}=\frac{1-\nu}{1+\nu}\left(\frac{\rho^{2}}{2}-\zeta^{2}\right)+\log \rho, & \hat{\Psi}_{1}=\hat{\Psi}_{2}=0, \\
\hat{\Psi}_{s}=-\frac{1}{1+\nu} \zeta, \\
v=\frac{1-\nu}{1+\nu} \rho+\frac{1}{\rho}, \quad v_{\theta}=0, & v_{z}=-\frac{2 \nu}{1+\nu} \zeta, \\
\tau_{r r}=1-\frac{1}{\rho^{2}}, & \tau_{\theta \theta}=1+\frac{1}{\rho^{2}}, \\
\tau_{z z}=\tau_{\theta z}=\tau_{z r}=\tau_{r \theta}=0 .
\end{array}
$$

Solution $\AA^{\prime \prime}$ (Plane-Strain Solution for Case 2).

$$
\begin{aligned}
\hat{\Phi}=\frac{1}{2}\left(\rho^{2}+\frac{1}{\rho^{2}}\right) \cos 2 \theta, & \hat{\Psi}_{1}=-\frac{1}{\rho} \cos \theta, \\
\hat{\Psi}_{2} & =\frac{1}{\rho} \sin \theta, \quad \hat{\Psi}_{3}=0,
\end{aligned}
$$

12 See, for example, Timoshenko-Goodier [15], for a derivation of these classical solutions on the basis of the Airy stress function.

$$
\begin{aligned}
& v_{r}=\left[\rho+\frac{4(1-\nu)}{\rho}-\frac{1}{\rho^{3}}\right] \cos 2 \theta, \\
& v_{\theta}=-\left[\rho+\frac{2(1-2 \nu)}{\rho}+\frac{1}{\rho^{3}}\right] \sin 2 \theta, \quad v_{z}=0, \\
& \tau_{r r}=\left(1-\frac{4}{\rho^{2}}+\frac{3}{\rho^{4}}\right) \cos 2 \theta, \\
& \tau_{s z}=-\frac{4 \nu}{\rho^{2}} \cos 2 \theta, \quad \tau_{\theta z}=-\left(1+\frac{3}{\rho^{4}}\right) \cos 2 \theta, \\
& \tau_{r \theta}=-\left(1+\frac{2}{\rho^{2}}-\frac{3}{\rho^{4}}\right) \sin 2 \theta,
\end{aligned}
$$

The plane-strain solution $\hat{S}^{\prime \prime}$ satisfies the elastostatic field equations, conforms to the loading condition (11) for Case 2, and meets all of the boundary conditions (4) and (5) with the exception of the requirement $\sigma_{33}=0$ on $\Pi$. This requirement is fulfilled by $\dot{S}^{\prime \prime}$ if and only if $\nu=0$, in which instance $S^{\prime \prime}$ is identical with $S^{\prime \prime}$. We now set

$$
S^{\prime \prime}=\check{S}^{\prime \prime}+\bar{S}
$$

and call $\bar{S}$, so defined, the solution to the "residual problem" of plane strain for Case 2. It is clear from (25) and the remarks preceding (25) that this residual problem is governed by the boundary conditions

$$
\begin{array}{r}
\tau_{r r}=\tau_{r \theta}=\tau_{r z}=0 \text { on } \rho=1(0 \leq \zeta<\infty), \\
\tau_{z r}=\tau_{z \theta}=0, \quad \tau_{z z}=\frac{2(2-\alpha)}{\rho^{2}} \cos 2 \theta \text { on } \zeta=0 \\
(1 \leq \rho<\infty),
\end{array}
$$

and the regularity conditions

$$
\tau_{r r}, \tau_{\theta \theta}, \tau_{z z}, \tau_{\theta z}, \tau_{s r}, \tau_{r \theta} \rightarrow 0 \text { as } \rho \rightarrow \infty \quad(0 \leq \zeta<\infty) \text {, }
$$

all of which must hold identically for $0 \leq \theta<2 \pi$.

Bearing in mind (12) and (25), we note that the determination of the solution $S$ to the original problem has been reduced to the task of constructing stress functions $\hat{\Phi}$ and $\hat{\Psi}_{i}$, harmonic in the interior of $R$, that generate-in the sense of (19) - a stress distribution satisfying (26), (27), and (28).

\section{Reduction of Residual Problem to a One-Dimensional Integral Equation}

Our next objective is to construct stress functions suited for the solution of the residual problem. To this end, guided by the $\theta$-dependence of $\tau_{z z}(\rho, \theta, 0)$ in (27) and by the manner in which $\theta$ enters (19), we set

$$
\begin{aligned}
\hat{\Phi}(\rho, \theta, \zeta) & =\varphi(\rho, \zeta) \cos 2 \theta \\
\hat{\Psi}_{1}(\rho, \theta, \zeta) & =\chi(\rho, \zeta) \cos \theta \\
\hat{\Psi}_{2}(\rho, \theta, \zeta) & =-\chi(\rho, \zeta) \sin \theta \\
\hat{\Psi}_{3}(\rho, \theta, \zeta) & =\psi(\rho, \zeta) \cos 2 \theta .
\end{aligned}
$$

On defining an auxiliary function $\omega$ through

$$
\omega=\varphi+\rho \chi+\zeta \psi,
$$

we infer from (29) and (17), (18), and (19) that now

$$
\begin{aligned}
& v_{r}=\left[\frac{\partial \omega}{\partial \rho}-2 \alpha \chi\right] \cos 2 \theta, \\
& v_{\theta}=\left[-\frac{2 \omega}{\rho}+2 \alpha \chi\right] \sin 2 \theta, \\
& v_{z}=\left[\frac{\partial \omega}{\partial \zeta}-2 \alpha \psi\right] \cos 2 \theta,
\end{aligned}
$$




$$
\begin{aligned}
\tau_{r r}= & {\left[\frac{\partial^{2} \omega}{\partial \rho^{2}}+(\alpha-2)\left(\frac{\partial \psi}{\partial \zeta}-\frac{\chi}{\rho}\right)\right.} \\
& \left.-(\alpha+2) \frac{\partial \chi}{\partial \rho}\right] \cos 2 \theta, \\
\tau_{\theta \theta}= & {\left[\frac{1}{\rho} \frac{\partial \omega}{\partial \rho}-\frac{4 \omega}{\rho^{2}}+(\alpha-2)\left(\frac{\partial \psi}{\partial \zeta}+\frac{\partial \chi}{\partial \rho}\right)\right.} \\
\tau_{z z}= & {\left[\frac{\partial^{2} \omega}{\partial \zeta^{2}}+(\alpha-2)\left(\frac{\partial \chi}{\partial \rho}-\frac{\chi}{\rho}\right)\right.} \\
\rho & \left.-(\alpha+2) \frac{\partial \psi}{\partial \zeta}\right] \cos 2 \theta
\end{aligned}
$$

$\tau_{\theta z}=\left[-\frac{2}{\rho} \frac{\partial \omega}{\partial \zeta}+\alpha \frac{\partial \chi}{\partial \zeta}+2 \alpha \frac{\psi}{\rho}\right] \sin 2 \theta$

$\tau_{\text {er }}=\left[\frac{\partial^{2} \omega}{\partial \rho \partial \zeta}-\alpha \frac{\partial \chi}{\partial \zeta}-\alpha \frac{\partial \psi}{\partial \rho}\right] \cos 2 \theta$,

$\tau_{r \theta}=\left[-2 \frac{\partial}{\partial \rho}\left(\frac{\omega}{\rho}\right)+\alpha\left(\frac{\partial \chi}{\partial \rho}+\frac{\chi}{\rho}\right)\right] \sin 2 \theta$.

Further, in view of (20), the stress functions $\Phi, \Psi_{i}$ determined by (29) and (15) are harmonic in the interior of $R$ if and only if the functions $\varphi, \chi, \psi$ on $(1, \infty) \times(0, \infty)$ satisfy

$$
\nabla_{1}^{2} \chi=0, \quad \nabla_{2}^{2} \varphi=\nabla_{2}^{2} \psi=0,
$$

where

$$
\nabla_{n}^{2}=\frac{\partial^{2}}{\partial \rho^{2}}+\frac{1}{\rho} \frac{\partial}{\partial \rho}+\frac{\partial^{2}}{\partial \zeta^{2}}-\frac{n^{2}}{\rho^{2}} \quad(n=1,2) .
$$

Separation of variables applied to the equation

$$
\nabla_{n}{ }^{2} F=0 \quad(n=1,2)
$$

at once yields the real-valued product solutions

$$
\begin{aligned}
& F(\rho, \zeta)=\left[J_{n}(\gamma \rho) \text { or } Y_{n}(\gamma \rho)\right] \\
& \times[\exp (\gamma \zeta) \text { or } \exp (-\gamma \zeta)] \text {, } \\
& F(\rho, \zeta)=\left[I_{n}(\gamma \rho) \text { or } K_{n}(\gamma \rho)\right][\cos (\gamma \zeta) \text { or } \sin (\gamma \zeta)] \text {, }
\end{aligned}
$$

in which $J_{n}, Y_{n}$ and $I_{n}, K_{n}$ are, respectively, the ordinary and the modified Bessel functions of the first and second kind, of order $n$, while $\gamma$ is an arbitrary nonnegative constant. For future reference, we also note that if $F$ satisfies (35), the same is true of

$$
F^{*}=\rho \frac{\partial F}{\partial \rho}+\zeta \frac{\partial F}{\partial \zeta} .
$$

This observation enables one to deduce from (36) solutions of (35) that are not obtainable by separation of variables.

We now seek to determine functions $\varphi, \chi, \psi$ that satisfy (33) and generate - in the sense of (32) - a stress field meeting the first two of the boundary conditions (27), i.e., giving rise to vanishing shearing tractions on $\zeta=0$; in addition, the resulting stress field must tend to zero as $\rho \rightarrow \infty$, in accordance with (28), and is to remain bounded as $\zeta \rightarrow \infty$. With this purpose in mind, we note first from (30) and (32) that $\tau_{z r}, \tau_{z \theta}$ are both identically zero at $\zeta=0$ if $\varphi, \chi$ are even functions of $\zeta$, while $\psi$ is odd in $\zeta$, and also if

$$
\frac{\partial \varphi}{\partial \zeta}=(\alpha-1) \psi, \quad \chi=0
$$

${ }^{13}$ Recall that the harmonicity of a function $H$ implies that of $\mathbf{x} \cdot \nabla H$, if $\mathbf{x}$ is the position vector.
In view of (36) and because of the regularity requirements ${ }^{14}$ mentioned before, we are thus led to set

$$
\begin{aligned}
& \varphi=\varphi_{1}+(\alpha-1) \varphi_{2}, \\
& \chi=\chi_{1}, \\
& \psi=\psi_{1}+\frac{\partial \varphi_{2}}{\partial \zeta^{\prime}},
\end{aligned}
$$

witk

$$
\begin{aligned}
& \varphi_{1}(\rho, \zeta)=\int_{0}^{\infty} A^{*}(\gamma) K_{2}(\gamma \rho) \cos (\gamma \zeta) d \gamma, \\
& \chi_{1}(\rho, \zeta)=\int_{0}^{\infty} B^{*}(\gamma) K_{1}(\gamma \rho) \cos (\gamma \zeta) d \gamma, \\
& \psi_{1}(\rho, \zeta)=\int_{0}^{\infty} C^{*}(\gamma) K_{2}(\gamma \rho) \sin (\gamma \zeta) d \gamma,
\end{aligned}
$$

in which $A^{*}, B^{*}, C^{*}$ are as yet arbitrary weight functions of the integration parameter $\gamma$.

It is apparent from (40), (30), and (32) that the contributions of $\varphi_{1}, \chi_{1}, \psi_{1}$ to all but the leading term in $\tau_{r r}(1, \theta, \zeta), \tau_{r \theta}(1, \theta, \zeta)$, and $\tau_{r z}(1, \theta, \zeta)$ take the form of Fourier integrals, whose respective integrands involve $\zeta$ only through the factor $\cos (\gamma \zeta)$ or sin $(\gamma \zeta)$. In contrast, the terms contributed through $\omega$ fail to exhibit such a structure. To remove this deficiency, which would prevent us from taking advantage of the Fourier-transform inversion theorem in coping with the boundary conditions (26), we replace the definition of $\varphi_{1}$ in (40) by

$$
\begin{aligned}
& \varphi_{1}(\rho, \zeta)=\int_{0}^{\infty}\left\{A^{*}(\gamma) K_{2}(\gamma \rho) \cos (\gamma \zeta)\right. \\
& \left.\quad+C^{*}(\gamma)\left[\rho K_{2}{ }^{\prime}(\gamma \rho) \cos (\gamma \zeta)-\zeta K_{2}(\gamma \rho) \sin (\gamma \zeta)\right]\right\} d \gamma .
\end{aligned}
$$

The integrand in (41) is an even function of $\zeta$ that possesses the required regularity as $\rho \rightarrow \infty$ and satisfies (33), as is clear from (36) and (37). Further, with this choice of $\varphi_{1}$, and with $\chi_{1}, \psi_{1}$ defined as in (40), one now has

$$
\begin{aligned}
\omega_{1}(\rho, \zeta) \equiv \varphi_{1}(\rho, \zeta)+\rho \chi_{1}(\rho, \zeta)+\zeta \psi_{1}(\rho, \zeta) & \\
= & \int_{0}^{\infty}\left[A^{*}(\gamma) K_{2}(\gamma \rho)+B^{*}(\gamma) \rho K_{1}(\gamma \rho)\right. \\
& \left.\quad+C^{*}(\gamma) \rho K_{2}{ }^{\prime}(\gamma \rho)\right] \cos (\gamma \zeta) d \gamma
\end{aligned}
$$

so that our immediate task has been accomplished.

We have yet to dispose of $\varphi_{2}$ in (39). The following choice of $\varphi_{2}$ is admissible by virtue of the first of (36); it is motivated by the form of the boundary condition on $\tau_{z s}$ in (27), as will become clear later on. We take

$$
\varphi_{2}(\rho, \zeta)=\int_{0}^{\infty} D^{*}(\gamma) \Omega_{2}(\gamma, \rho) \exp (-\gamma \zeta) d \gamma,
$$

where

$$
\Omega_{2}(\gamma, \rho)=Y_{2}^{\prime}(\gamma) J_{2}(\gamma \rho)-J_{2}^{\prime}(\gamma) Y_{2}(\gamma \rho),
$$

while $D^{*}$ is yet another initially arbitrary function of the integration parameter. To shorten future results, it is convenient to define a new quadruplet of weight functions $A, B, C$, and $D$ through

$$
\begin{aligned}
A^{*}=A-\frac{2}{\alpha} C, & B^{*}=-\frac{\gamma}{\alpha} C, \\
C^{*} & =\gamma B-\frac{\gamma}{\alpha} C, \quad D^{*}=-\gamma^{2} D .
\end{aligned}
$$

In view of (39), the last two of (40), and (41), (43), (45), as well as

14 Recall that $I_{n}(\gamma \rho)$ becomes unbounded as $\rho \rightarrow \infty$.

15 Throughout this paper, $K_{n}{ }^{\prime}, J_{n}{ }^{\prime}$, and $Y_{n}{ }^{\prime}$ denote the first derivatives of the corresponding Bessel functions. 
the recurrence relation expressing $K_{1}$ in terms of $K_{2}$ and $K_{2}^{\prime}$, we arrive at the final choice of $\varphi, \chi, \psi$ :

$$
\begin{aligned}
\varphi(\rho, \zeta)= & \int_{0}^{\infty}\left\{\left[A(\gamma)-\frac{2}{\alpha} C(\gamma)\right] K_{2}(\gamma \rho) \cos (\gamma \zeta)\right. \\
+ & {\left[B(\gamma)-\frac{1}{\alpha} C(\gamma)\right]\left[\gamma \rho K_{2}^{\prime}(\gamma \rho) \cos (\gamma \zeta)\right.} \\
& \left.-\gamma \zeta K_{2}(\gamma \rho) \sin (\gamma \zeta)\right] \\
& \left.+(1-\alpha) \gamma^{2} D(\gamma) \Omega_{2}(\gamma, \rho) \exp (-\gamma \zeta)\right\} d \gamma
\end{aligned}
$$

$\chi(\rho, \zeta)=\frac{1}{\alpha} \int_{0}^{\infty} C(\gamma)\left[\frac{2}{\rho} K_{2}(\gamma \rho)\right.$

$$
\left.+\gamma K_{2}{ }^{\prime}(\gamma \rho)\right] \cos (\gamma \zeta) d \gamma
$$

$$
\begin{array}{r}
\psi(\rho, \zeta)=\int_{0}^{\infty}\left\{\left[B(\gamma)-\frac{1}{\alpha} C(\gamma)\right] \gamma K_{2}(\gamma \rho) \sin (\gamma \zeta)\right. \\
\left.+\gamma^{3} D(\gamma) \Omega_{2}(\gamma, \rho) \exp (-\gamma \zeta)\right\} d \gamma
\end{array}
$$

The four weight functions $A, B, C$, and $D$ in (46) are to be determined from the remaining four boundary conditions, i.e., in accordance with (26) and the last of (27). We therefore substitute formally from (46) into the first and the last two of (32). In this manner using the recurrence relations for $K_{n}$, the modified Bessel equation, as well as the identity

$$
\Omega_{2}(\gamma, 1)=\frac{2}{\pi \gamma}
$$

and adopting the auxiliary notation

$$
K(\gamma)=\gamma \frac{K_{2}{ }^{\prime}(\gamma)}{K_{2}(\gamma)}
$$

we find that $\tau_{r r}(1, \theta, \zeta), \tau_{r \theta}(1, \theta, \zeta)$, and $\tau_{r z}(1, \theta, \zeta)$ vanish identically, provided, for $0 \leq \zeta<\infty$,

$$
\begin{gathered}
\int_{0}^{\infty}\left\{A(\gamma)\left[\gamma^{2}+4-K(\gamma)\right]\right. \\
+B(\gamma)\left[(\alpha-1) \gamma^{2}-4+\left(\gamma^{2}+4\right) K(\gamma)\right] \\
\left.-2 C(\gamma)\left[\gamma^{2}+2+K(\gamma)\right]\right\} K_{2}(\gamma) \cos (\gamma \zeta) d \gamma \\
=\frac{2}{\pi} \int_{0}^{\infty} \gamma D(\gamma)[4(\alpha-1-\gamma \zeta) \\
\left.+\gamma^{2}(\gamma \zeta-1)\right] \exp (-\gamma \zeta) d \gamma
\end{gathered}
$$

$\int_{0}^{\infty}\left\{2 A(\gamma)[1-K(\gamma)]+2 B(\gamma)\left[-\left(\gamma^{2}+4\right)+K(\gamma)\right]\right.$

$$
\begin{aligned}
& \left.+C(\gamma)\left[\gamma^{2}+4+2 K(\gamma)\right]\right\} K_{2}(\gamma) \cos (\gamma \zeta) d \gamma \\
& =\frac{4}{\pi} \int_{0}^{\infty} \gamma D(\gamma)(\alpha-1-\gamma \zeta) \exp (-\gamma \zeta) d \gamma
\end{aligned}
$$

$\int_{0}^{\infty}\left\{A(\gamma) K(\gamma)+B(\gamma)\left[\gamma^{2}+4+\alpha K(\gamma)\right]\right.$

$$
-2 C(\gamma)[1+K(\gamma)]\} \gamma K_{2}(\gamma) \sin (\gamma \zeta) d \gamma=0
$$

Similarly, $\tau_{s z}(\rho, \theta, 0)$ is found to satisfy the last of (27) if, for $1 \leq$ $\rho<\infty$,

$$
\begin{array}{r}
\int_{0}^{\infty} \gamma^{4} D(\gamma) \Omega_{2}(\gamma, \rho) d \gamma=\frac{2(2-\alpha)}{\rho^{2}} \\
+\int_{0}^{\infty}\left\{[A(\gamma)+(\alpha+2) B(\gamma)-2 C(\gamma)] K_{2}(\gamma \rho)\right. \\
\left.+B(\gamma) \gamma \rho K_{2}{ }^{\prime}(\gamma \rho)\right\} \gamma^{2} d \gamma .
\end{array}
$$

16 See [16], p. 79 , No. 28 .
Equations (49)-(52) constitute a system of four simultaneous linear integral equations for the unknown functions $A, B, C$, and $D$. With a view toward reducing this system to a single integral equation, we recall first that the inversion theorem for the Fourier cosine and sine transforms ${ }^{17}$ furnishes the identity

$f(\eta)=\frac{2}{\pi} \int_{0}^{\infty} \int_{0}^{\infty} f(\gamma)\left\{\begin{array}{l}\cos (\gamma \zeta) \cos (\eta \zeta) \\ \sin (\gamma \zeta) \sin (\eta \zeta)\end{array}\right\} d \gamma d \zeta$

$$
(0<\eta<\infty),
$$

valid for every $f$ which is suitably well-behaved on $[0, \infty)$. Further, we note the particular Fourier cosine transforms ${ }^{18}$

$$
\begin{aligned}
& \int_{0}^{\infty} \gamma \exp (-\gamma \zeta) \cos (\eta \zeta) d \zeta=g(\eta / \gamma) \\
& \int_{0}^{\infty} \gamma(1+\gamma \zeta) \exp (-\gamma \zeta) \cos (\eta \zeta) d \zeta=2 g^{2}(\eta / \gamma)
\end{aligned}
$$

where

$$
g(x)=\left(1+x^{2}\right)^{-1} .
$$

We now multiply (49), (50) by $(2 / \pi) \cos (\eta \zeta),(51)$ by $(2 / \pi) \sin$ $(\eta \zeta)$, integrate the resulting equations with respect to $\zeta$ over the range $[0, \infty)$, and use $(53)-(55)$ to obtain

$$
\begin{gathered}
\begin{array}{c}
A(\eta)\left[\eta^{2}+4-K(\eta)\right] \\
+B(\eta)\left[(\alpha-1) \eta^{2}-4+\left(\eta^{2}+4\right) K(\eta)\right] \\
-2 C(\eta)\left[\eta^{2}+2+K(\eta)\right]
\end{array} \\
=\frac{8}{\pi^{2} K_{2}(\eta)} \int_{0}^{\infty} D(\gamma)\left[2 \alpha-\left(\eta^{2}+4\right) g(\eta / \gamma)\right] g(\eta / \gamma) d \gamma, \\
2 A(\eta)[1-K(\eta)]+2 B(\eta)\left[-\left(\eta^{2}+4\right)+K(\eta)\right] \\
\quad+C(\eta)\left[\eta^{2}+4+2 K(\eta)\right] \\
=\frac{8}{\pi^{2} K_{2}(\eta)} \int_{0}^{\infty} D(\gamma)[\alpha-2 g(\eta / \gamma)] g(\eta / \gamma) d \gamma,
\end{gathered}
$$

$A(\eta) K(\eta)+B(\eta)\left[\eta^{2}+4+\alpha K(\eta)\right]$

$$
-2 C(\eta)[1+K(\eta)]=0 .
$$

Equations (56) are three simultaneous linear algebraic equations in $A, B$, and $C$; their solution, after a convenient change of the dummy variables, is given by

$$
\begin{aligned}
A(\gamma)= & \frac{16 \alpha}{\pi^{2} K_{2}(\gamma) \Delta(\gamma)}\left[f_{1}(\gamma)+f_{4}(\gamma)\right] D_{1}(\gamma) \\
& -\frac{8}{\pi^{2} K_{2}(\gamma) \Delta(\gamma)}\left\{\left[\gamma^{2}+4+\alpha K(\gamma)\right] f_{3}(\gamma)\right. \\
& \left.\quad-4 \alpha f_{2}(\gamma)[K(\gamma)+1]\right\} D_{2}(\gamma) \\
B(\gamma)=\frac{16 \alpha}{\pi^{2} K_{2}(\gamma) \Delta(\gamma)} f_{2}(\gamma) D_{1}(\gamma) & +\frac{8}{\pi^{2} K_{2}(\gamma) \Delta(\gamma)} K(\gamma) f_{3}(\gamma) D_{2}(\gamma) \\
C(\gamma)=\frac{8 \alpha \quad}{\pi^{2} K_{2}(\gamma) \Delta(\gamma)} f_{1}(\gamma) D_{1}(\gamma) & +\frac{16 \alpha}{\pi^{2} K_{2}(\gamma) \Delta(\gamma)} K(\gamma) f_{2}(\gamma) D_{2}(\gamma)
\end{aligned}
$$

where

$$
\begin{aligned}
& D_{1}(\gamma)=\int_{0}^{\infty} D(\xi) g(\gamma / \xi) d \xi, \\
& D_{2}(\gamma)=\int_{0}^{\infty} D(\xi) g^{2}(\gamma / \xi) d \xi,
\end{aligned}
$$

${ }^{17}$ See, for example, [17 ], p. 17 .

${ }^{18}$ See [18], p. 14. 


$$
\begin{aligned}
f_{1}(\gamma) & =\gamma^{2}\left[\gamma^{2}+4-K^{2}(\gamma)\right]+3 \alpha K^{2}(\gamma), \\
f_{2}(\gamma) & =\gamma^{2}-\left(\gamma^{2}+3\right) K(\gamma), \\
f_{3}(\gamma) & =\left(\gamma^{2}+2\right)\left(\gamma^{2}+6\right)-2 \gamma^{2} K(\gamma), \\
f_{4}(\gamma)= & \left.\quad \gamma^{2}+3\right)\left(\gamma^{2}+4\right) \\
\Delta(\gamma) & =\left[\gamma^{2}+4-K^{2}(\gamma)\right] f_{3}(\gamma) \\
+ & \alpha\left[-4 \gamma^{2}+8\left(\gamma^{2}+3\right) K(\gamma)-\gamma_{2} K^{2}(\gamma)-3 \alpha K(\gamma), 6 K^{3}(\gamma)\right]
\end{aligned}
$$

Turning to (52), we cite the following inversion identity, which is a modified form of Weber's integral theorem ${ }^{19}$ and holds true for any function $f$ sufficiently regular on $[0, \infty)$ and every $n(n=$ $0,1,2, \ldots)$ :

$$
\begin{aligned}
f(\eta)\left\{\left[J_{n}^{\prime}(\eta)\right]^{2}+\right. & {\left.\left[Y_{n}^{\prime}(\eta)\right]^{2}\right\} } \\
& =\int_{1}^{\infty} \int_{0}^{\infty} \gamma \rho f(\gamma) \Omega_{n}(\gamma, \rho) \Omega_{n}(\eta, \rho) d \gamma d \rho,
\end{aligned}
$$

where

$$
\Omega_{n}(\gamma, \rho)=Y_{n}^{\prime}(\gamma) J_{n}(\gamma \rho)-J_{n}^{\prime}(\gamma) Y_{n}(\gamma \rho)
$$

A proof of (60), restricted to $n=0$, was given by Blenkarn [20], ${ }^{20}$ who used the inversion formula (60) with this value of $n$ in connection with a rotationally symmetric half-space problem described in the Introduction. A proof applicable to arbitrary integral values of $n$ is readily obtained along the lines of Watson's [12] proof of Weber's theorem. For the problem we are considering, the relevant value of $n$ is two in view of $(52), \Omega_{2}(\gamma, \rho) \exp$ $(-\gamma \zeta)$ being a solution of (35) with $n=2$. Indeed, as is now evident, the choice of $\varphi_{2}$ in (43) was motivated by the fact that (60) enables one to reduce (52) - with $A, B, C$ given by (57) - to a Fredholm equation for the unknown weight function $D$.

To effect the reduction just alluded to, we shall need to make use of the definite integrals

$$
\begin{gathered}
\int_{1}^{\infty} \frac{1}{\rho} \Omega_{2}(\eta, \rho) d \rho=\frac{4}{\pi \eta^{3}}, \\
\int_{1}^{\infty} \rho \Omega_{2}(\eta, \rho) K_{2}(\gamma \rho) d \rho=-\frac{2 \gamma}{\pi \eta^{3}} K_{2}^{\prime}(\gamma) g(\gamma / \eta), \\
\int_{1}^{\infty} \gamma \rho^{2} \Omega_{2}(\eta, \rho) K_{2}^{\prime}(\gamma \rho) d \rho=\frac{2}{\pi \eta^{3}}\left\{-\left(\gamma^{2}+4\right) K_{2}(\gamma)\right. \\
\left.+2 \gamma K_{2}^{\prime}(\gamma)[1-g(\gamma / \eta)]\right\} g(\gamma / \eta),
\end{gathered}
$$

in which $g$ is once again given by (55). Equations (62) may be deduced from the indefinite integral

$\int \rho \Omega_{2}(\eta, \rho) K_{2}(\gamma \rho) d \rho$

$$
=\frac{\rho}{\gamma^{2}+\eta^{2}}\left[\Omega_{2}(\eta, \rho) \frac{\partial}{\partial \rho} K_{2}(\gamma \rho)-K_{2}(\gamma \rho) \frac{\partial}{\partial \rho} \Omega_{2}(\eta, \rho)\right],
$$

which can be verified directly by differentiation and an appeal to the ordinary and the modified Bessel equation. Thus the second of (62) is obtained by evaluating (63) for the appropriate limits of integration, taking account of (61) and (47); the first of (62) follows from the second if one multiplies the latter by $\gamma^{2}$ and then passes to the limit as $\gamma \rightarrow 0$; finally, differentiation of the second of (62) with respect to $\gamma$, in conjunction with the modified Bessel equation, yields the third.

Now multiply (52) by $\rho \Omega_{2}(\eta, \rho)$, integrate the resulting identity with respect to $\rho$ over the range $[1, \infty)$, and invoke $(60),(62)$ to confirm that

\footnotetext{
19 See Watson [12], p. 468, and Titchmarsh [19].

${ }^{20}$ For a condensed published version of [20], see Blenkarn and Wilhoit [13].
}

$$
\begin{aligned}
D(\eta) \eta^{3}\left\{\left[J_{2}^{\prime}(\eta)\right]^{2}+\left[Y_{2}^{\prime}(\eta)\right]^{2}\right\}=\frac{8(2-\alpha)}{\pi \eta^{3}} \\
-\frac{2}{\pi \eta^{3}} \int_{0}^{\infty}\left\{A(\gamma) K(\gamma)+B(\gamma)\left[\gamma^{2}+4+\alpha K(\gamma)\right]\right. \\
-2 C(\gamma) K(\gamma)+2 B(\gamma) K(\gamma) g(\gamma / \eta)\} g(\gamma / \eta) \gamma^{2} K_{2}(\gamma) d \gamma .
\end{aligned}
$$

Substituting for $A, B$, and $C$ from (57) into (64) and letting

$$
Z(\eta)=\left[J_{2}^{\prime}(\eta)\right]^{2}+\left[Y_{2}^{\prime}(\eta)\right]^{2},
$$

we arrive at the integral equation

$$
D(\eta) \eta^{6} Z(\eta)=\frac{8(2-\alpha)}{\pi}+\int_{0}^{\infty} D(\xi) L(\xi, \eta) d \xi,
$$

whose symmetric kernel $L$ is defined on $[0, \infty) \times[0, \infty)$ through

$$
\begin{aligned}
& L(\xi, \eta)=-\frac{32}{\pi^{3}} \int_{0}^{\infty} \frac{\gamma^{2}}{\Delta(\gamma)} g(\gamma / \xi) g(\gamma / \eta)\left\{\alpha f_{1}(\gamma)\right. \\
&+2 \alpha[g(\gamma / \xi)+g(\gamma / \eta)] K(\gamma) f_{2}(\gamma) \\
&\left.+g(\gamma / \xi) g(\gamma / \eta) K^{2}(\gamma) f_{3}(\gamma)\right\} d \gamma .
\end{aligned}
$$

The functions $g$ and $f_{1}, f_{2}, f_{3}, \Delta$ appearing in (67) are those previously defined in (55) and (59), respectively. Equation (66) is a Fredholm equation of the second kind for the weight function $D$.

\section{Displacements and Stresses of the Solution to the Residual Problem: Numerical Evaluations and Results}

The stress functions (46) involve the four weight functions $A$, $B, C$, and $D$, and (57) relate $A, B, C$ to $D$. Accordingly, (30)-(32) permit us to express the displacements and stresses of the solution $\bar{S}$ to the residual problem in terms of the solution $D$ of the integral equation (66) and known functions. We now record the results reached in this manner and for this purpose adopt the additional notation

$$
\begin{gathered}
\Omega_{2}{ }^{\prime}(\gamma, \rho)=Y_{2}{ }^{\prime}(\gamma) J_{2}{ }^{\prime}(\gamma \rho)-J_{2}{ }^{\prime}(\gamma) Y_{2}{ }^{\prime}(\gamma \rho), \\
G(\gamma, \rho)=\frac{K_{2}(\gamma \rho)}{K_{2}(\gamma)}, \quad G^{\prime}(\gamma, \rho)=\frac{\rho K_{2}{ }^{\prime}(\gamma \rho)}{K_{2}{ }^{\prime}(\gamma)} .
\end{gathered}
$$

$$
\begin{aligned}
& \begin{array}{c}
\text { Displacements of Solution } \bar{S} . \\
\frac{v_{r}(\rho, \theta, \zeta)}{\cos 2 \theta}=\int_{0}^{\infty} D(\eta)\left[(1-\alpha+\eta \zeta) \eta^{3} \Omega_{2}^{\prime}(\eta, \rho)\right. \\
\left.\quad \times \exp (-\eta \zeta)+w_{r}(\eta, \rho, \zeta)\right] d \eta
\end{array} \\
& \begin{array}{c}
\frac{v_{\theta}(\rho, \theta, \zeta)}{\sin 2 \theta}=\int_{0}^{\infty} D(\eta)\left[2(\alpha-1-\eta \zeta) \frac{\eta^{2}}{\rho} \Omega_{2}(\eta, \rho)\right. \\
\left.\quad \times \exp (-\eta \zeta)+w_{\theta}(\eta, \rho, \zeta)\right] d \eta, \\
\frac{v_{z}(\rho, \theta, \zeta)}{\cos 2 \theta}=\int_{0}^{\infty} D(\eta)\left[-(\alpha+\eta \zeta) \eta^{3} \Omega_{2}(\eta, \rho)\right. \\
\left.\times \exp (-\eta \zeta)+w_{z}(\eta, \rho, \zeta)\right] d \eta,
\end{array}
\end{aligned}
$$

where

$$
\begin{aligned}
& w_{r}(\eta, \rho, \zeta)=\frac{8}{\pi^{2}} \int_{0}^{\infty} \frac{\cos (\gamma \zeta)}{\rho \Delta(\gamma)} \\
& \times\left[2 \alpha g ( \gamma / \eta ) \left\{G(\gamma, \rho)\left[\left(\gamma^{2} \rho^{2}+4\right) f_{2}(\gamma)-2 f_{1}(\gamma)\right]\right.\right. \\
& \left.+G^{\prime}(\gamma, \rho) K(\gamma) f_{4}(\gamma)\right\}+g^{2}(\gamma / \eta) K(\gamma) \\
& \quad \times\left\{G(\gamma, \rho)\left[\left(\gamma^{2} \rho^{2}+4\right) f_{3}(\gamma)-8 \alpha f_{2}(\gamma)\right]\right. \\
& \left.\left.+G^{\prime}(\gamma, \rho)\left[-\left(\gamma^{2}+4+\alpha K(\gamma)\right) f_{3}(\gamma)+4 \alpha f_{2}(\gamma)\right]\right\}\right] d \gamma
\end{aligned}
$$

$w_{\theta}(\eta, \rho, \zeta)=\frac{16}{\pi^{2}} \int_{0}^{\infty} \frac{\cos (\gamma \zeta)}{\rho \Delta(\gamma)}\left[\alpha g(\gamma / \eta)\left\{-2 G(\gamma, \rho) f_{4}(\gamma)\right.\right.$ 


$$
\begin{gathered}
\left.+G^{\prime}(\gamma, \rho) K(\gamma)\left[f_{1}(\gamma)-2 f_{2}(\gamma)\right]\right\} \\
+g^{2}(\gamma / \eta)\left\{G(\gamma, \rho)\left[\left(\gamma^{2}+4+\alpha K(\gamma)\right) f_{3}(\gamma)-4 \alpha f_{2}(\gamma)\right]\right. \\
\left.\left.\quad+G^{\prime}(\gamma, \rho) K^{2}(\gamma)\left[-f_{3}(\gamma)+2 \alpha f_{2}(\gamma)\right]\right\}\right] d \gamma \\
w_{z}(\eta, \rho, \zeta)=\frac{8}{\pi^{2}} \int_{0}^{\infty} \frac{\gamma \sin (\gamma \zeta)}{\Delta(\gamma)} \\
\times\left[-2 \alpha g(\gamma / \eta)\left\{G(\gamma, \rho)\left[f_{4}(\gamma)+2 \alpha f_{2}(\gamma)\right]\right.\right. \\
\left.\quad+G^{\prime}(\gamma, \rho) K(\gamma) f_{2}(\gamma)\right\}+g^{2}(\gamma / \eta) \\
\times\left\{G ( \gamma , \rho ) \left[\left(\gamma^{2}+4-\alpha K(\gamma)\right) f_{3}(\gamma)\right.\right.
\end{gathered}
$$

Stresses of Solution $\bar{S}$.

$$
\begin{aligned}
& \frac{\tau_{r r}(\rho, \theta, \zeta)}{\cos 2 \theta}=\int_{0}^{\infty} D(\eta) {\left[\left\{\left[\frac{4}{\rho^{2}}(1-\alpha+\eta \zeta)\right.\right.\right.} \\
&\left.\left.+(1-\eta \zeta) \eta^{2}\right] \Omega_{2}(\eta, \rho)+(\alpha-1-\eta \zeta) \frac{\eta}{\rho} \Omega_{2}{ }^{\prime}(\eta, \rho)\right\} \eta^{2} \\
& \\
&\left.\times \exp (-\eta \zeta)+t_{r r}(\eta, \rho, \zeta)\right] d \eta
\end{aligned}
$$

$$
\begin{aligned}
\frac{\tau_{\theta \theta}(\rho, \theta, \zeta)}{\cos 2 \theta}=\int_{0}^{\infty} D(\eta) & \left\{\left[\frac{4}{\rho^{2}}(\alpha-1-\eta \zeta)\right.\right. \\
\left.+(2-\alpha) \eta^{2}\right] \Omega_{2}(\eta, \rho)+ & \left.(1-\alpha+\eta \zeta) \frac{\eta}{\rho} \Omega_{2}{ }^{\prime}(\eta, \rho)\right\} \eta^{2} \\
& \left.\times \exp (-\eta \zeta)+t_{\theta \theta}(\eta, \rho, \zeta)\right] d \eta
\end{aligned}
$$

$$
\begin{array}{r}
\frac{\tau_{z x}(\rho, \theta, \zeta)}{\cos 2 \theta}=\int_{0}^{\infty} D(\eta)\left[(1+\eta \zeta) \eta^{4} \Omega_{2}(\eta, \rho) \exp (-\eta \zeta)\right. \\
\left.+t_{z z}(\eta, \rho, \zeta)\right] d \eta
\end{array}
$$

$$
\begin{aligned}
\frac{\tau_{\theta z}(\rho, \theta, \zeta)}{\sin 2 \theta}=\int_{0}^{\infty} D(\eta)\left[\frac{2 \eta^{4} \zeta}{\rho} \Omega_{2}(\eta, \rho)\right. & \exp (-\eta \zeta) \\
& \left.+t_{\theta z}(\eta, \rho, \zeta)\right] d \eta
\end{aligned}
$$$$
\frac{\tau_{s r}(\rho, \theta, \zeta)}{\cos 2 \theta}=\int_{0}^{\infty} D(\eta)\left[-\eta^{5} \zeta \Omega_{2}^{\prime}(\eta, \rho) \exp (-\eta \zeta)\right.
$$$$
\left.+t_{z r}(\eta, \rho, \zeta)\right] d \eta
$$

$$
\begin{array}{r}
\frac{\tau_{r \theta}(\rho, \theta, \zeta)}{\sin 2 \theta}=\int_{0}^{\infty} D(\eta)\left\{2 ( 1 - \alpha + \eta \zeta ) \left[\frac{1}{\rho^{2}} \Omega_{2}(\eta, \rho)\right.\right. \\
\left.\left.-\frac{\eta}{\rho} \Omega_{2}^{\prime}(\eta, \rho)\right] \eta^{2} \exp (-\eta \zeta)+t_{r \theta}(\eta, \rho, \zeta)\right\} d \eta
\end{array}
$$

where

$$
\begin{aligned}
& t_{r r}(\eta, \rho, \zeta)=\frac{8}{\pi^{2}} \int_{0}^{\infty} \frac{\cos (\gamma \zeta)}{\rho^{2} \Delta(\gamma)} \\
& \quad \times\left\{2 \alpha g ( \gamma / \eta ) \left[G ( \gamma , \rho ) \left\{\left[(\alpha-1) \gamma^{2} \rho^{2}-4\right] f_{2}(\gamma)\right.\right.\right. \\
& \left.+2 f_{1}(\gamma)+\left(\gamma^{2} \rho^{2}+4\right) f_{4}(\gamma)\right\} \\
& \left.+G^{\prime}(\gamma, \rho) K(\gamma)\left[\left(\gamma^{2} \rho^{2}+4\right) f_{2}(\gamma)-2 f_{1}(\gamma)-f_{4}(\gamma)\right]\right] \\
& +g^{2}(\gamma / \eta)\left[G ( \gamma , \rho ) \left\{-\left[\left(\gamma^{2} \rho^{2}+4\right)\left(\gamma^{2}+4+K(\gamma)\right)\right.\right.\right. \\
& \left.+4 \alpha K(\gamma)] f_{3}(\gamma)+4 \alpha\left[\gamma^{2} \rho^{2}+4+2 K(\gamma)\right] f_{2}(\gamma)\right\} \\
& +G^{\prime}(\gamma, \rho) K(\gamma)\left\{\left[\left(\gamma^{2} \rho^{2}+4+\alpha\right) K(\gamma)+\gamma^{2}+4\right] f_{3}(\gamma)\right. \\
& t_{\theta 9}(\eta, \rho, \zeta)=\frac{8}{\pi^{2}} \int_{0}^{\infty} \frac{\cos (\gamma \zeta)}{\rho^{2} \Delta(\gamma)} \\
& \quad \times\left\{2 \alpha g ( \gamma / \eta ) \left[G ( \gamma , \rho ) \left\{\left[(\alpha-1) \gamma^{2} \rho^{2}+4\right] f_{2}(\gamma)\right.\right.\right.
\end{aligned}
$$

$$
\begin{aligned}
& \left.-2 f_{1}(\gamma)-4 f_{4}(\gamma)\right\}+G^{\prime}(\gamma, \rho) K(\gamma)\left[-4 f_{2}(\gamma)\right. \\
& \left.\left.+2 f_{1}(\gamma)+f_{4}(\gamma)\right]\right]+g^{2}(\gamma / \eta)\left[G ( \gamma , \rho ) \left\{\left[(\alpha-1) \gamma^{2} \rho^{2} K(\gamma)\right.\right.\right. \\
& \left.+4\left(\gamma^{2}+4+K(\gamma)+\alpha K(\gamma)\right)\right] f_{3}(\gamma) \\
& \left.-8 \alpha[K(\gamma)+2] f_{2}(\gamma)\right\}+G^{\prime}(\gamma, \rho) K(\gamma) \\
& \times\left\{-\left[\gamma^{2}+4+4 K(\gamma)+\alpha K(\gamma)\right] f_{3}(\gamma)\right. \\
& \left.\left.\left.+4 \alpha[2 K(\gamma)+1] f_{2}(\gamma)\right\}\right]\right\} d \gamma, \\
& t_{z z}(\eta, \rho, \zeta)=\frac{8}{\pi^{2}} \int_{0}^{\infty} \frac{\gamma^{2} \cos (\gamma \zeta)}{\Delta(\gamma)} \\
& \times\left[-2 \alpha g(\gamma / \eta)\left\{G(\gamma, \rho)\left[(\alpha+2) f_{2}(\gamma)+f_{4}(\gamma)\right]\right.\right. \\
& \left.+G^{\prime}(\gamma, \rho) K(\gamma) f_{2}(\gamma)\right\}+g^{2}(\gamma / \eta)\left\{G ( \gamma , \rho ) \left[\left(\gamma^{2}+4\right.\right.\right. \\
& \left.-2 K(\gamma)) f_{3}(\gamma)-4 \alpha f_{2}(\gamma)\right] \\
& \left.\left.-G^{\prime}(\gamma, \rho) K^{2}(\gamma) f_{3}(\gamma)\right\}\right] d \gamma, \\
& t_{\theta x}(\eta, \rho, \zeta)=\frac{8}{\pi^{2}} \int_{0}^{\infty} \frac{\gamma \sin (\gamma \zeta)}{\rho \Delta(\gamma)} \\
& \times\left[\alpha g ( \gamma / \eta ) \left\{4 G(\gamma, \rho)\left[\alpha f_{2}(\gamma)+f_{4}(\gamma)\right]\right.\right. \\
& \left.+G^{\prime}(\gamma, \rho) K(\gamma)\left[4 f_{2}(\gamma)-f_{1}(\gamma)\right]\right\} \\
& +2 g^{2}(\gamma / \eta)\left\{G(\gamma, \rho)\left[-\left(\gamma^{2}+4\right) f_{3}(\gamma)+4 \alpha f_{2}(\gamma)\right]\right. \\
& \left.\left.+G^{\prime}(\gamma, \rho) K^{2}(\gamma)\left[f_{3}(\gamma)-\alpha f_{2}(\gamma)\right]\right\}\right] d \gamma, \\
& t_{z r}(\eta, \rho, \zeta)=\frac{8}{\pi^{2}} \int_{0}^{\infty} \frac{\gamma \sin (\gamma \zeta)}{\rho \Delta(\gamma)} \\
& \times\left[2 \alpha g ( \gamma / \eta ) \left\{G ( \gamma , \rho ) \left[-\left(\gamma^{2} \rho^{2}+4\right) f_{2}(\gamma)\right.\right.\right. \\
& \left.\left.+f_{1}(\gamma)\right]-G^{\prime}(\gamma, \rho) K(\gamma)\left[\alpha f_{2}(\gamma)+f_{4}(\gamma)\right]\right\} \\
& +g^{2}(\gamma / \eta) K(\gamma)\left\{G(\gamma, \rho)\left[-\left(\gamma^{2} \rho^{2}+4\right) f_{3}(\gamma)+4 \alpha f_{2}(\gamma)\right]\right. \\
& \left.\left.+G^{\prime}(\gamma, \rho)\left[\left(\gamma^{2}+4\right) f_{3}(\gamma)-4 \alpha f_{2}(\gamma)\right]\right\}\right] d \gamma, \\
& t_{r \theta}(\eta, \rho, \zeta)=\frac{8}{\pi^{2}} \int_{0}^{\infty} \frac{\cos (\gamma \zeta)}{\rho^{2} \Delta(\gamma)} \\
& \times\left\{\alpha g ( \gamma / \eta ) \left\{G ( \gamma , \rho ) \left[-4\left(\gamma^{2} \rho^{2}+4\right) f_{2}(\gamma)\right.\right.\right. \\
& \left.+\left(\gamma^{2} \rho^{2}+8\right) f_{1}(\gamma)+4 f_{4}(\gamma)\right]+2 G^{\prime}(\gamma, \rho) K(\gamma)\left[2 f_{2}(\gamma)\right. \\
& \left.\left.-f_{1}(\gamma)-2 f_{4}(\gamma)\right]\right\}+2 g^{2}(\gamma / \eta)[G(\gamma, \rho) \\
& \times\left\{-\left[\left(\gamma^{2} \rho^{2}+4+\alpha\right) K(\gamma)+\gamma^{2}+4\right] f_{3}(\gamma)\right. \\
& \left.+\alpha\left[\gamma^{2} \rho^{2} K(\gamma)+4+8 K(\gamma)\right] f_{2}(\gamma)\right\} \\
& +G^{\prime}(\gamma, \rho) K(\gamma)\left\{\left[\gamma^{2}+4+K(\gamma)+\alpha K(\gamma)\right] f_{3}(\gamma)\right. \\
& \left.\left.\left.-2 \alpha[2+K(\gamma)] f_{2}(\gamma)\right\}\right]\right\} d \gamma .
\end{aligned}
$$

For the sake of convenience, we refer once more to the definitions of all auxiliary symbols appearing in $S$ : The functions $G, G^{\prime}, \Omega_{2}$, $\Omega_{2}^{\prime}$ are accounted for by (44) and (68); $K$ is given by (48), and $f_{1}, f_{2}, f_{3}, f_{4}, \Delta$ by (59); $g$ and $\alpha$ are defined by (55) and (17), respectively. Note that $\bar{S}$ depends on Poisson's ratio in a complicated fashion. Thus the parameter $\alpha \equiv 2(1-\nu)$ enters (69-72) not only explicitly but also through $\Delta, f_{1}$, and $f_{4}$, as well as through $D$ since the kernel (67) of $(66)$ contains $\alpha, f_{1}$, and $\Delta$.

Once (66) has been solved for $D$, the desired displacements and stresses of the solution $\bar{S}$ to the residual problem are completely determined by (69)-(72). In view of the unwieldiness of the kernel (67), it was not feasible to treat (66) analytically. For this reason, the integral equation (66) was solved numerically on an IBM 704 electronic computer for the values of Poisson's ratio $\nu=1 / 4,1 / 2(\alpha=3 / 2,1)$, the solution corresponding to $\nu=0$ being known in advance. ${ }^{21}$

In order to keep the present paper to a reasonable length, the details of these and of subsequent numerical computations will have to be omitted here. A comprehensive account of the numerical work carried out, and of the extensive supplementary

${ }^{21}$ Recall that $\bar{S}$ vanishes identically when $\nu=0$. 


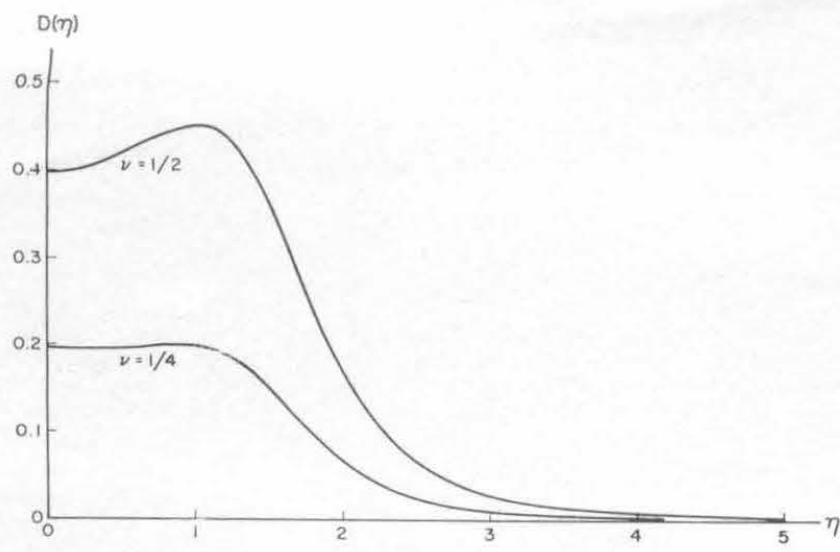

Fig. 2 Solution of infegral equation for $D(\eta)$

analytical work performed to accelerate the convergence of the solution, may be found in a separate report [21].

Since the derivation of $\bar{S}$ in the preceding section involves various purely formal manipulations whose validity depends upon the anticipated nature of the initially unknown weight functions $A, B, C$, and $D$, some remarks concerning the a posteriori verification of the solution to the residual problem are in order. The numerical solution $D$ of the Fredholm equation $(66)$ is depicted in Fig. 2. Further, a plot of $\log (D(\eta))$ versus $\log \eta$ indicates the asymptotic behavior

$$
D(\eta)=c(\nu) \eta^{-4}+o\left(\eta^{-4}\right) \text { as } \eta \rightarrow \infty,
$$

with $c(1 / 4) \doteq 0.322, c(1 / 2) \doteq 1.26$. On the other hand, equation (73), together with the continuity of $D$ on $[0, \infty)$, insures the required convergence of the improper integrals in (69)-(72) and entitles one to differentiate the displacements and stresses of $\bar{S}$ under the respective integral signs. Such differentiations, in turn, enable one to confirm that $\bar{S}$ indeed satisfies the cylindrical counterpart of the field equations (2) and (3). Also, a tedious but straightforward computation based on (69)-(72) verifies that $\bar{S}$ meets all of the boundary conditions (26), the first two of (27), as well as (28), for every $D$ that possesses the foregoing regularity properties. Finally, for every such $D$, the displacements (69) and the stresses (71) are found to vanish in the limit as $\zeta \rightarrow \infty$, so that, by (25), the solution $S^{\prime \prime}$ for Case 2 approaches the associated plane-strain solution $S^{\prime \prime}$ in this limit.

We have yet to confirm the last of the boundary conditions (27), i.e.,

$\tau_{z z}(\rho, \theta, 0)=\frac{2(2-\alpha)}{\rho^{2}} \cos 2 \theta \quad(1 \leq \rho<\infty, \quad 0 \leq \theta<2 \pi)$,

the fulfillment of which depends evidently upon the specific values of $D$. Accordingly, the verification of (74) supplies an essential check on the accuracy of the numerical solution of (66) and thereby gives an indication of the accuracy to be expected of the entire solution to the problem under consideration. The typical difficulties encountered in the numerical evaluation of the stress field appropriate to $\bar{S}$ are illustrated in especially severe form by those attending the computation of $\tau_{z z}(\rho, \theta, 0)$. To convey an idea of these complications, we first recall from (71) that

$$
\begin{aligned}
\frac{\tau_{z z}(\rho, \theta, \zeta)}{\cos 2 \theta}=\int_{0}^{\infty} D(\eta)(1+\eta \zeta) & \eta^{4} \Omega_{2}(\eta, \rho) \exp (-\eta \zeta) d \eta \\
& +\int_{0}^{\infty} D(\eta) t_{z z}(\eta, \rho, \zeta) d \eta
\end{aligned}
$$

where $t_{z z}$ is itself an improper integral and is given by the third of (72). The first integrand in the right-hand member of (75), because of the factor $\exp (-\eta \zeta)$, decays rapidly as $\eta \rightarrow \infty$ when $\zeta>0$. For $\zeta=0$, however, this integrand is an oscillatory function of slowly decreasing amplitude. The improper integral representing $t_{z z}$ is also found to be poorly convergent. Since an accurate knowledge of $t_{z}(\eta, \rho, 0)$ is an essential prerequisite for the performance of the second integration required by (75), it was necessary to examine the asymptotic behavior of the integrand of $t_{z z}$ for large values of $\gamma$ and to remove in closed form-in terms of sine and cosine integrals-certain contributions to $t_{z x}(\eta$, $\rho, 0)$ that impede its direct evaluation. An additional difficulty arises from the fact that the integral representation (75) for $\tau_{z z}$ is discontinuous along the edge $\rho=1, \zeta=0$. Indeed, one finds from (75) that

$$
\tau_{z z}(1+, \theta, 0+)=\tau_{z z}(1, \theta, 0)+4 c(\nu) / \pi .
$$

This discontinuous behavior is reflected in the slow convergence of (75) near $\rho=1, \zeta=0$. Nevertheless, as is apparent from Table 1, the error inherent in the computed values of $\tau_{z z}(\rho, \theta, 0)$ is insignificant also in the vicinity of $\rho=1$. It will be observed, however, that the deviations of the numerical from the theoretical values of $\tau_{z z}(\rho, \theta, 0)$ increase in magnitude as $\rho$ approaches unity. Finally, it should be emphasized that, for reasons already mentioned, the numerical results for the stresses away from the plane boundary, i.e., at $\zeta>0$, are apt to be appreciably more accurate than those summarized in Table 1.

\section{Table 1 Check on boundary condition for $\sigma_{z z}$ in residual problem}

\begin{tabular}{ccccc}
\multicolumn{5}{c}{$\sigma_{z z}(\rho, \theta, 0) / \sigma \cos 2 \theta$} \\
\hline \multicolumn{1}{c}{$\nu=1 / 4$} & \multicolumn{2}{c}{$\nu=1 / 2$} \\
\hline$\rho$ & $\begin{array}{c}\text { Numerical } \\
\text { value }\end{array}$ & $\begin{array}{c}\text { Theoretical } \\
\text { value }\end{array}$ & $\begin{array}{c}\text { Numerical } \\
\text { value }\end{array}$ & $\begin{array}{c}\text { Theoretical } \\
\text { value }\end{array}$ \\
\hline 1 & 1.00218 & 1.00000 & 2.01167 & 2.00000 \\
1.02 & 0.95576 & 0.96117 & 1.89914 & 1.92234 \\
1.1 & 0.82730 & 0.82645 & 1.65466 & 1.65289 \\
1.2 & 0.69578 & 0.69444 & 1.39262 & 1.38889 \\
1.4 & 0.51100 & 0.51020 & 1.02146 & 1.02041 \\
1.6 & 0.39070 & 0.39063 & 0.78148 & 0.78125 \\
1.8 & 0.30879 & 0.30864 & 0.61737 & 0.61728 \\
2 & 0.25011 & 0.25000 & 0.49982 & 0.50000 \\
4 & 0.06228 & 0.06250 & 0.12432 & 0.12500 \\
6 & 0.02706 & 0.02778 & 0.05381 & 0.05556 \\
8 & 0.01523 & 0.01563 & 0.03120 & 0.03125 \\
10 & 0.00903 & 0.01000 & 0.02027 & 0.02000
\end{tabular}

Of primary physical concern is the variation with $\zeta$ of the nonvanishing stresses along the boundary of the hole $\rho=1$ and the radial variation of the normal displacement along the plane boundary $\zeta=0$. A detailed account of the numerical evaluation of $\tau_{z z}(1, \theta, \zeta), \tau_{\theta z}(1, \theta, \zeta), \tau_{\theta \theta}(1, \theta, \zeta)$, and $v_{z}(\rho, \theta, 0)$ is included in [21]. The results obtained are plotted in Figs. $3-6$ for Case 2, which corresponds to the state of pure shear (11) at infinity and represents the basic nontrivial loading case. Thus the present numerical results are based on the solution

$$
S^{\prime \prime}=\dot{S}^{\prime \prime}+\bar{S},
$$

in which $\dot{S}^{\prime \prime}$ is the plane-strain solution (24) associated with Case 2 , whereas $\bar{S}$ is the solution of the residual problem given by (69)(72). Analogous numerical results for the general loading conditions (6) are immediately deducible from those presented here by means of (12) and (22).

Each of the diagrams to be discussed includes three curves, corresponding to the values of Poisson's ratio $\nu=1 / 2, \nu=1 / 4$, and $\nu=0$. In this connection, we recall that $\dot{S}^{\prime \prime}$ is the exact solution for Case 2 when $\nu=0$, so that

$$
S^{\prime \prime}=\AA^{\prime \prime} \text { for } \nu=0 .
$$

Also, since all components of $\bar{S}$ tend to zero as $\zeta \rightarrow \infty$, one has

$$
S^{\prime \prime} \rightarrow \dot{S}^{\prime \prime} \quad \text { as } \zeta \rightarrow \infty,
$$

regardless of the particular value of Poisson's ratio. In view of 
the fact that the residual tractions $\tau_{z z}(\rho, \theta, 0)$ to which $\AA^{\prime \prime}$ gives rise on $\zeta=0$ are self-equilibrated, the conclusion (79) confirms an expectation suggested by $(77)$ and an intuitive appeal to Saint-Venant's principle. ${ }^{22}$

Figs. 3 and 4 show the $\zeta$-dependence at the cylindrical boundary of the transverse normal stress $\tau_{z z}$ and the transverse shear stress $\tau_{\theta z}$, respectively. The variation with $\zeta$ of the circumferential normal stress $\tau_{\theta \theta}$ at $\rho=1$ is given in Fig. 5. These graphs display clearly the three-dimensional boundary-layer effect that constitutes the main objective of the present paper. As is apparent from Fig. 3, when $\nu>0, \tau_{z z}$ departs radially from its respective plane-strain values (dashed lines) in the vicinity of $\zeta=0$ but is already virtually indistinguishable from $\tau_{z z}$ of solution $\AA^{\prime \prime}$ at $\zeta=3$. Similarly, the shear stress $\tau_{\theta z}$, which vanishes identically in $S^{\prime \prime}$, according to Fig. 4 attains its maximum magnitude at approximately $\zeta=0.35$ and decays rapidly as $\zeta$ increases beyond this value; at $\zeta=3$, the magnitude of $\tau_{\theta z}$ is less than 3 percent of the maximum magnitude of $\tau_{\theta \theta}$. The relevant departures of $\tau_{\theta \theta}$ from its plane-strain values are confined to an even thinner boundary layer. Thus Fig. 5 reveals that for $\nu=1 / 2$ the planestrain solution overestimates the magnitude of $\tau_{\theta \theta}$ up to $\zeta \doteq 0.45$, the actual value of $\left|\tau_{\theta \theta}(1, \theta, 0) / \cos 2 \theta\right|$ being 2.35 as compared to the value of four predicted by $\dot{S}^{\prime \prime}$. In contrast, the magnitude of $\tau_{\theta \theta}$ in $S^{\prime \prime}$ is only slightly larger than in $\AA^{\prime \prime}$ for $\zeta>0.45$ when $\nu=$

${ }^{22}$ Note, however, that the plane region $I$ over which $\AA^{\prime \prime}$ violates the boundary conditions $\tau_{z z}(\rho, \theta, 0)=0$ is unbounded.

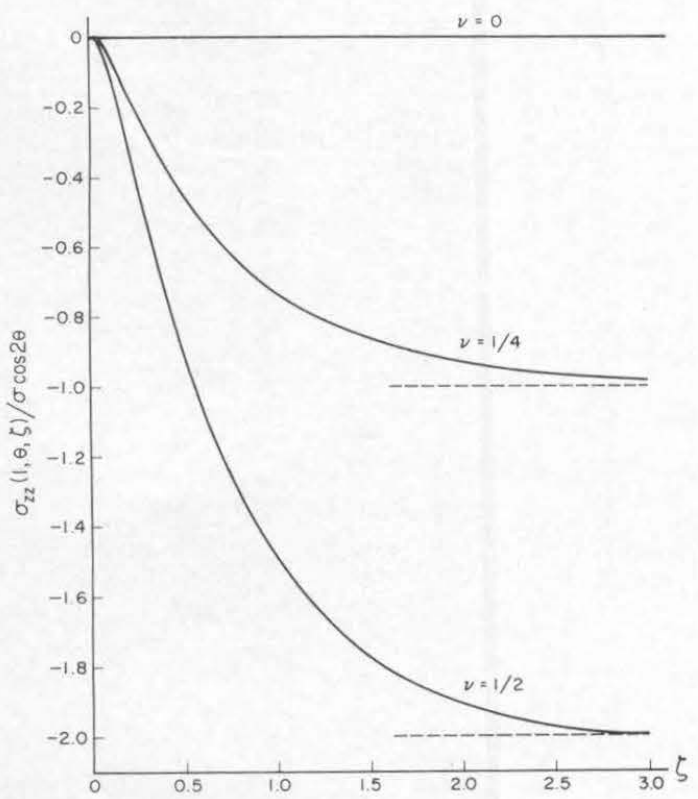

Fig. 3 Dependence of $\sigma_{z z}$ on $\zeta$ at $\rho=1$

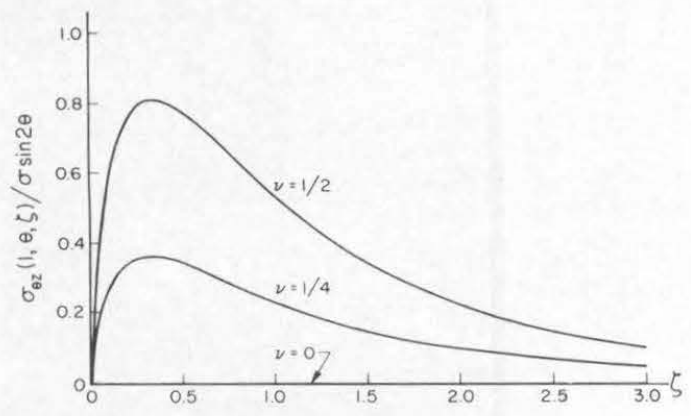

Fig. 4 Dependence of $\sigma_{\theta z}$ on $\zeta$ at $\rho=1$
1/2. Finally, Fig. 6 depicts the dependence upon $\rho$ of the normal displacement $v_{2}$ at the plane boundary $\zeta=0$. This displacement component vanishes identically in the plane-strain solution. As was to be anticipated, all of the three-dimensional effects under discussion are highly sensitive to changes in Poisson's ratio and become more pronounced at larger values of this parameter.

\section{References}

1 E. Reissner, "On the Calculation of Three-Dimensional Corrections for the Two-Dimensional Theory of Plane Stress," Proceedings of the Fifteenth Semi-Annual Eastern Photoelasticity Conference, 1942.

2 A. E. Green, "The Elastic Equilibrium of Isotropic Plates and Cylinders," Proceedings of the Royal Society of London, London, England, Series A, vol. 195, 1949, p. 533.

3 E. L. Reiss and S. Locke, "On the Theory of Plane Stress," Quarterly of A pplied Mathematics, vol. 19, no. 3, 1961, p. 195.

$4 \mathrm{~K}$. O. Friedrichs, The Edge Effect in the Bending of Plates, Reissner Anniversary Volume, Edwards, Ann Arbor, Mich., 1949.

5 E. Sternberg and R. Muki, "Note on the Expansion in Powers of Poisson's Ratio of Solutions in Elastostatics," Arch. Rat. Mech. Anal., vol. 3, no. 3, 1959, p. 229.

6 A. E. Green and T. J. Willmore, "Three-Dimensional Stress Systems in Isotropic Plates II, Proceedings of the Royal Society of London, London, England, Series A, vol. 193, 1948, p. 229.

7 G. Kirseh, "Die Theorie der Elastizität und die Bedurfnisse

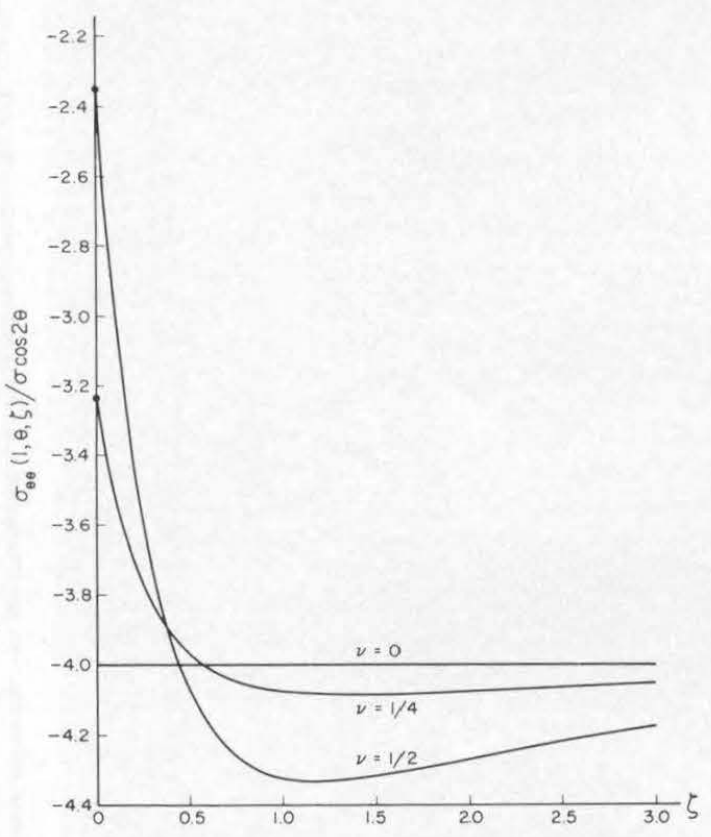

Fig. 5 Dependence of $\sigma_{\theta \theta}$ on $\zeta$ at $\rho=1$

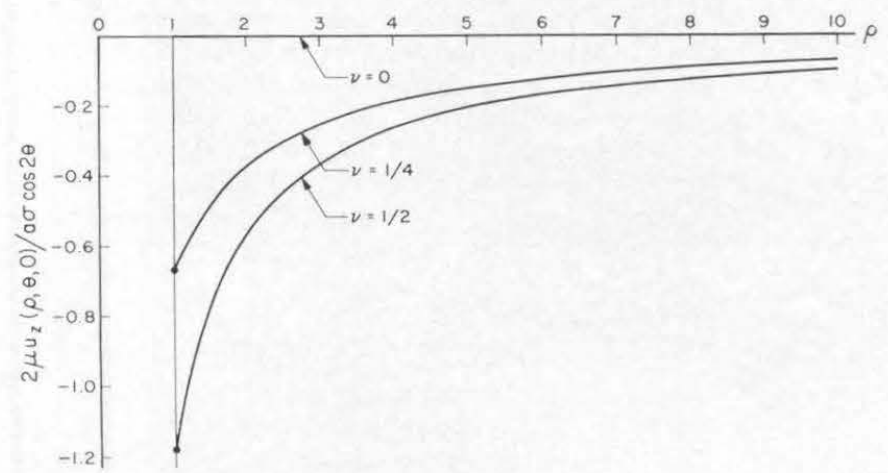

Fig. 6 Dependence of $v_{z}$ on $\rho$ at $\zeta=0$ 
der Festigkeitslehre," Zentralblatt Verein Deutscher Ingenieure, vol. 42,1898 , p. 797 .

8 A. E. Green, "Three-Dimensional Stress Systems in Isotropic Plates, I," Transactions of the Royal Society of London, London, England, Series A, vol. 240, 825, 1948, p. 561.

9 E. Sternberg and M. A. Sadowsky, "Three-Dimensional Solution for the Stress Concentration Around a Circular Hole in a Plate of Arbitrary Thickness," Journal of Applied Mechanics, vol. 16, Trans. ASME, vol. 71, 1949, pp. 27-38.

10 J. B. Alblas, "Theorie van de driedimensionale spanningstoestand in een doorboovde plaat," dissertation, Technische Hogeschool Delft, H. J. Paris, Amsterdam, The Netherlands, 1957.

11 E. L. Reiss, "Extension of an Infinite Plate With a Circular Hole, Journal of the Society for Industrial and Applied Mathematics, vol. 11 , no. 4,1963 , p. 840 .

12 G. N. Watson, A Treatise on the Theory of Bessel Functions, second edition, Cambridge University Press, Cambridge, England, 1948.

13 K. A. Blenkarn and J. C. Wilhoit, "Stresses Due to a Band of Normal Stress at the Entrance of a Circular Hole," Journal of Applied Mechanics, vol. 29, Trans. ASME, vol. 84, Series E, 1962, pp. 647-650.

14 M. A. Sadowsky and Eli Sternberg, "Stress Concentration
Around a Triaxial Ellipsoidal Cavity," Journal of Applied MeCHANICs, vol. 16, Trans. ASME, vol. 71, 1949, pp. 149-157.

15 S. Timoshenko and J. N. Goodier, Theory of Elasticity, second edition, McGraw-Hill Book Company, Inc., New York, N. Y., 1951.

16 Tables of Integral Transforms, vol. 2, Bateman Manuscript Project, MeGraw-Hill Book Company, Ine., New York, N. Y., 1954.

17 I. N. Sneddon, Fourier Transforms, MeGraw-Hill Book Company, Inc., New York, N. Y., 1951.

18 Tables of Integral Transforms, vol. 1, Bateman Manuscript Project, McGraw-Hill Book Company, Inc., New York, N. Y., 1954.

19 E. C. Titehmarsh, "Weber's Integral Theorem," Proceedings of the London Mathematical Society, Series 2, vol. 22, 1923, p. 15.

20 K. A. Blenkarn, "Fourier Integral Solutions to Radially Symmetric Elasticity Problems," dissertation, Rice Institute, Houston, Texas, 1960.

21 C. K. Youngdahl and E. Sternberg, "Three-Dimensional Stress Concentration Around a Cylindrical Hole in a Semi-Infinite Elastic Body," Research and Development Report, Argonne National Laboratory, Argonne, IIl., ANL 7097, 1965.

22 H. Weber, "Über die Darstellung willkürlicher Functionen durch Bessel'sche Functionen," Mathematische Annalen, vol. 6, 1873, p. 146. 